\title{
PARACOMPACTNESS OF PIXLEY-ROY HYPERSPACES. I
}

\author{
HIDENORI TANAKA
}

\begin{abstract}
In this paper we will study the paracompactness of Pixley-Roy hyperspaces of products. We will prove that if $\mathscr{S}[X]$ is paracompact (resp. hereditarily paracompact), then $\mathscr{F}\left[X^{2}\right]$ is paracompact (resp. hereditarily paracompact). This is the positive answer to T. Przymusinski's problem.
\end{abstract}

1. Introduction. Throughout this paper we will assume that $X$ is a $T_{1}$-space. This paper continues the study of the paracompactness of Pixley-Roy hyperspaces initiated in [8]. Recall that for each space $X$, the space $\mathscr{F}[X]$, called the Pixley-Roy hyperspace over $X$, defined by C. Pixley and P. Roy in [5], is the set of all nonempty finite subsets of $X$ with the topology generated by the sets of the form $[F, U]=\{G$ $\in \mathscr{F}[X]: F \subset G \subset U\}$, where $F \in \mathscr{F}[X]$ and $U$ is an open neighborhood of $F$ in $X$. E. K. van Douwen pointed out that $\mathscr{F}[X]$ is a zero-dimensional Tychonoff space [2]. For each $n \in N$, let $\mathscr{F}_{n}[X]=\{F \in \mathscr{F}[X]: \operatorname{card}(F)<n\}$. We consider $\mathscr{F}_{n}[X]$ with the subspace topology of $\mathscr{F}[X]$ for each $n \in N$.

T. Przymusiński characterized the paracompactness (resp. hereditary paracompactness) of Pixley-Roy hyperspaces as follows [6, Theorems 2.1, 2.2].

THEOREM 1. The following conditions are equivalent:

(a) $\mathscr{F}[X]$ is paracompact;

(b) $\mathscr{F}[X]^{n}$ is paracompact for each $n \in N$;

(c) for every nonempty finite subset $F$ of $X$ one can choose an open neighborhood $U_{F}$ so that the inclusions $F \subset U_{H}$ and $H \subset U_{F}$ imply $F \cap H \neq \varnothing$.

THEOREM 2. The following conditions are equivalent.

(a) $\mathscr{F}[X]$ is hereditarily paracompact;

(b) $\mathscr{F}[X]^{n}$ is hereditarily paracompact for each $n \in N$;

(c) for every nonempty finite subset $F$ of $X$ one can choose an open neighborhood $U_{F}$ so that the inclusions $F \subset U_{H}$ and $H \subset U_{F}$ imply $F \subset H$ or $H \subset F$.

So T. Przymusiński raised the following problem [6, Problem 2].

Problem. Suppose that $\mathscr{F}[X]$ is paracompact. Is $\mathscr{F}\left[X^{2}\right]$ paracompact?

The purpose of this paper is to answer this problem affirmatively. Furthermore we will obtain the hereditarily paracompact case. We will prove the following.

THEOREM 3. Suppose that $\mathscr{F}[X]$ is paracompact (resp. hereditarily paracompact). Then $\mathscr{F}\left[X^{2}\right]$ is paracompact (resp. hereditarily paracompact).

Received by the editors December 10, 1980.

1980 Mathematics Subject Classification. Primary S4B20, S4D18; Secondary S4E30, S4E35.

Key words and phrases. Paracompact, hereditarily paracompact, Pixley-Roy hyperepace, Moore space. 0002-9939/81/0000-1119/\$02.50 
Let $\mathscr{W}$ be a collection of subsets of $\mathscr{F}[X]$. We denote $\cup\{W: W \in W\}$ by $W^{*}$. By $X \oplus Y$ we mean the topological sum of spaces $X$ and $Y$. For undefined terminology, the reader is referred to [3]. For Pixley-Roy hyperspaces, the reader is referred to [2] and [6].

2. Proof of Theorem 3. The following lemma was proved by the author in [8].

LEMMA. Let $\mathbb{Q}$ be an open-and-closed subset of $\mathscr{F}[X]$ such that $\mathbb{Q} \supset \mathscr{F}_{n}[X]$. For each $F \in \mathscr{F}_{n+1}[X]-\mathcal{Q}$, let $\left\{W_{F}(x): x \in F\right\}$ be a collection of open subsets of $X$ such that:

(1) $x \in W_{F}(x)$ for each $x \in F$,

(2) $\left[F, \cup\left\{W_{F}(x): x \in F\right\}\right] \cap \mathbb{Q}=\varnothing$, and

(3) $\left[G, \cup\left\{W_{F}(x): x \in G\right\}\right] \subset \mathbb{Q}$ for each $G \subset F$ such that $\operatorname{card}(G)<n$.

If $\left[F, \cup\left\{W_{F}(x): x \in F\right\}\right] \cap\left[H, \cup\left\{W_{H}(y): y \in H\right\}\right] \neq \varnothing$ for some $F$ and $H$ in $\mathscr{F}_{n+1}[X]-\mathcal{Q}$, then each $W_{F}(x)$ contains exactly one element of $H$.

Proof of Theorem 3. Suppose that $\mathscr{F}[X]$ is paracompact. Then there exists a collection $\mathfrak{U}=\left\{U_{F}: F \in \mathscr{F}[X]\right\}$ of open subsets of $X$ satisfying the condition (c) of Theorem 1. Let

$$
\mho_{1}=\left\{\left[\{(x, y)\}, U_{\{x\}} \times U_{\{y\}}\right]:(x, y) \in X^{2}\right\} .
$$

Suppose that

$$
\left[\{(x, y)\}, U_{\{x\}} \times U_{\{y\}}\right] \cap\left[\{(z, w)\}, U_{\{z\}} \times U_{\{w\}}\right] \neq \varnothing
$$

for some $(x, y),(z, w) \in X^{2}$. Then $(x, y) \in U_{\{z\}} \times U_{\{w\}}$ and $(z, w) \in U_{\{x\}} \times$ $U_{\{y\}}$. Hence $x \in U_{\{z\}}, y \in U_{\{w\}}, z \in U_{\{x\}}$ and $w \in U_{\{y\}}$. Hence $x=z$ and $y=w$ by the property of $\mathfrak{u}$. Hence $(x, y)=(z, w)$. Hence $\mathscr{W}_{1}$ is pairwise disjoint in $\mathscr{F}\left[X^{2}\right]$. We have already obtained a collection $\left\{\mathscr{W}_{i}: i=1, \ldots, n\right\}$ of pairwise disjoint families of basic open subsets of $\mathscr{F}\left[X^{2}\right]$ such that:

(3) $\cup_{i=1}^{j} \mho_{i}^{*} \supset \mathscr{F}_{j}\left[X^{2}\right]$ for each $j<n$, and

$\left(4_{n}\right) \mho_{i}^{*} \cap \mathscr{W}_{j}^{*}=\varnothing$ for $i, j<n$ and $i \neq j$.

Let $\mathscr{Q}=\cup_{i=1}^{n} W_{i}^{*}$. Then $\mathscr{Q}$ is a closed subset of $\mathscr{F}\left[X^{2}\right]$ by Lemma 1 in [8] (or Lemma 2.3 in [6]). For each

$$
F=\left\{\left(x_{1}, y_{1}\right), \ldots,\left(x_{n}, y_{n}\right),\left(x_{n+1}, y_{n+1}\right)\right\} \in \mathscr{F}_{n+1}\left[X^{2}\right]-\mathcal{Q},
$$

let $F_{1}=\left\{x_{1}, \ldots, x_{n}, x_{n+1}\right\}$ and $F_{2}=\left\{y_{1}, \ldots, y_{n}, y_{n+1}\right\}$. For each $x \in F_{1}$, let $F_{1}^{x}=F_{1}-\{x\}$. For each $y \in F_{2}$, let $F_{2}^{y}=F_{2}-\{y\}$. For each $F$ in ( $\left.\dagger\right)$, there exist open neighborhoods $W_{F}\left(x_{i}\right)$ and $W_{F}\left(y_{i}\right)$ of $x_{i}$ and $y_{i}$, respectively, $i=1, \ldots, n$, $n+1$, such that:

(5) $\left[F, \cup_{i=1}^{n+1} W_{F}\left(x_{i}\right) \times W_{F}\left(y_{i}\right)\right] \cap \mathbb{Q}=\varnothing$;

(6) for every subset $G$ of $F$ such that $\operatorname{card}(G)<n,\left[G, \cup_{(x, y) \in G} W_{F}(x) \times W_{F}(y)\right]$ $\subset \mathbb{Q}$

(7) for every subset $H$ of $F_{1}, \cup_{x \in H} W_{F}(x) \subset U_{H}$;

(8) for every subset $I$ of $F_{2}, \cup_{y \in I} W_{F}(y) \subset U_{I}$;

(9) for each $x \in F_{1}, W_{F}(x) \cap F_{1}^{x}=\varnothing$; and

(10) for each $y \in F_{2}, W_{F}(y) \cap F_{2}^{y}=\varnothing$. 
For each $F$ in $(\dagger)$ let

$$
W(F)=\bigcup_{i=1}^{n+1} W_{F}\left(x_{i}\right) \times W_{F}\left(y_{i}\right) .
$$

Let

$$
\mho_{n+1}=\left\{[F, W(F)]: F \in \mathscr{F}_{n+1}\left[X^{2}\right]-\mathscr{Q}\right\} .
$$

We will prove that $\mathscr{W}_{n+1}$ is pairwise disjoint in $\mathscr{F}\left[X^{2}\right]$. Suppose that $[F, W(F)] \cap$ $[G, W(G)] \neq \varnothing$ for some

$$
\begin{gathered}
F=\left\{\left(x_{1}, y_{1}\right), \ldots,\left(x_{n}, y_{n}\right),\left(x_{n+1}, y_{n+1}\right)\right\}, \\
G=\left\{\left(z_{1}, w_{1}\right), \ldots,\left(z_{n}, w_{n}\right),\left(z_{n+1}, w_{n+1}\right)\right\} \in \mathscr{F}_{n+1}\left[X^{2}\right]-\mathcal{Q} .
\end{gathered}
$$

Then $F \subset W(G)$ and $G \subset W(F)$. By the Lemma, $W_{F}\left(x_{1}\right) \times W_{F}\left(y_{1}\right)$ contains an element of $G$. So we can suppose $\left(z_{1}, w_{1}\right) \in W_{F}\left(x_{1}\right) \times W_{F}\left(y_{1}\right)$ (if necessary, by reordering the $\left(z_{i}, w_{i}\right)$ 's). We will prove that $\left(x_{1}, y_{1}\right) \in W_{G}\left(z_{1}\right) \times W_{G}\left(w_{1}\right)$. Suppose that $\left(x_{1}, y_{1}\right) \notin W_{G}\left(z_{1}\right) \times W_{G}\left(w_{1}\right)$. By the Lemma, $W_{G}\left(z_{1}\right) \times W_{G}\left(w_{1}\right)$ contains an element of $F$. So we can suppose $\left(x_{2}, y_{2}\right) \in W_{G}\left(z_{1}\right) \times W_{G}\left(w_{1}\right)$. Then $\left(z_{1}, w_{1}\right) \notin$ $W_{F}\left(x_{2}\right) \times W_{F}\left(y_{2}\right)$. For if not, suppose $\left(z_{1}, w_{1}\right) \in W_{F}\left(x_{2}\right) \times W_{F}\left(y_{2}\right)$. By the Lemma,

$$
G-\left\{\left(z_{1}, w_{1}\right)\right\} \subset \bigcup_{i=3}^{n+1} W_{F}\left(x_{i}\right) \times W_{F}\left(y_{i}\right) .
$$

$\operatorname{But} \operatorname{card}\left(F-\left\{\left(x_{1}, y_{1}\right),\left(x_{2}, y_{2}\right)\right\}\right)=n-1$ and $\operatorname{card}\left(G-\left\{\left(z_{1}, w_{1}\right)\right\}\right)=n$. Hence there exists some $i(3<i<\mathrm{n}+1)$ such that $W_{F}\left(x_{i}\right) \times W_{F}\left(y_{i}\right)$ contains two distinct elements of $G$, which contradicts the Lemma. Hence $\left(z_{1}, w_{1}\right) \notin W_{F}\left(x_{2}\right) \times$ $W_{F}\left(y_{2}\right)$. We can suppose that $\left(z_{2}, w_{2}\right) \in W_{F}\left(x_{2}\right) \times W_{F}\left(y_{2}\right)$. By the same reason, $\left(x_{2}, y_{2}\right) \notin W_{G}\left(z_{2}\right) \times W_{G}\left(w_{2}\right)$. So we can suppose that $\left(x_{3}, y_{3}\right) \in W_{G}\left(z_{2}\right) \times W_{G}\left(w_{2}\right)$. We continue this process repeatedly. Then we obtain subsets $B=\left\{\left(x_{1}\right.\right.$, $\left.\left.y_{1}\right), \ldots,\left(x_{m}, y_{m}\right)\right\}$ and $C=\left\{\left(z_{1}, w_{1}\right), \ldots,\left(z_{m}, w_{m}\right)\right\}, m<n+1$, of $F$ and $G$, respectively, such that:

(11) $\left(z_{i}, w_{i}\right) \in W_{F}\left(x_{i}\right) \times W_{F}\left(y_{i}\right)$ for each $i<m$, and

(12) $\left(x_{i+1}, y_{i+1}\right) \in W_{G}\left(z_{i}\right) \times W_{G}\left(w_{i}\right)$ for each $i<m-1$ and $\left(x_{1}, y_{1}\right) \in W_{G}\left(z_{m}\right)$ $\times W_{G}\left(w_{m}\right)$.

Let $H=\left\{x_{1}, \ldots, x_{m}\right\}$ and $I=\left\{z_{1}, \ldots, z_{m}\right\}$. Then

$$
H \cup I \subset \bigcup_{i=1}^{m} W_{F}\left(x_{i}\right) \cap \bigcup_{i=1}^{m} W_{G}\left(z_{i}\right) .
$$

By (7), $H \cup I \subset U_{H} \cap U_{I}$. By the property of $\mathfrak{u}, H \cap I \neq \varnothing$. Hence $x_{i}=z_{j}$ for some $i, j<m$. By $(12),\left(x_{i}, y_{i}\right) \in W_{G}\left(z_{i-1}\right) \times W_{G}\left(w_{i-1}\right)$ (if $i=1$, then $\left(x_{1}, y_{1}\right) \in$ $\left.W_{G}\left(z_{m}\right) \times W_{G}\left(w_{m}\right)\right)$. Suppose that $x_{i} \neq z_{i-1}$. Then $z_{i-1} \neq z_{j}$. By (9), $W_{G}\left(z_{i-1}\right) \nexists z_{j}$, which is a contradiction. Hence $x_{i}=z_{i-1}$. Since $\left(z_{i-1}, w_{i-1}\right) \in W_{F}\left(x_{i-1}\right) \times$ $W_{F}\left(y_{i-1}\right)$, we similarly obtain that $x_{i-1}=z_{i-1}$. By (11) and (12), we can continue this process repea edly. Then we obtain $x_{1}=z_{1}=\cdots=x_{m}=z_{m}$. Similarly we obtain $y_{1}=w_{1}=\cdots=y_{m}=w_{m}$. Hence $\left(x_{1}, y_{1}\right)=\cdots=\left(x_{m}, y_{m}\right)$, which contradicts the fact that $F \in \mathscr{F}_{n+1}\left[X^{2}\right]$. Hence $\left(x_{1}, y_{1}\right) \in W_{G}\left(z_{1}\right) \times W_{G}\left(w_{1}\right)$. By (7) 
and (8), we obtain $x_{1}=z_{1}$ and $y_{1}=w_{1}$. Hence $\left(x_{1}, y_{1}\right)=\left(z_{1}, w_{1}\right)$. Similarly we obtain $\left(x_{i}, y_{i}\right)=\left(z_{i}, w_{i}\right)$ for each $i=2, \ldots, n, n+1$ (if necessary, by reordering the $\left(z_{i}, w_{i}\right)$ 's, $\left.i=2, \ldots, n, n+1\right)$. Hence $F=G$. Hence $W_{n+1}$ is pairwise disjoint in $\mathscr{F}\left[X^{2}\right]$. Clearly $\left\{\mho_{i}: i=1, \ldots, n, n+1\right\}$ satisfies conditions $\left(3_{n+1}\right)$ and $\left(4_{n+1}\right)$. Inductively we obtain a sequence $\left\{\mathscr{W}_{n}: n \in N\right\}$ of pairwise disjoint families of basic open subsets of $\mathscr{F}\left[X^{2}\right]$ such that:

(13) $\cup\left\{\mathscr{W}_{n}: n \in N\right\}$ covers $\mathscr{F}\left[X^{2}\right]$, and

(14) $\mathscr{W}_{i}^{*} \cap \mathscr{W}_{j}^{*}=\varnothing$ for $i, j \in N$ and $i \neq j$.

Let $W=\cup\left\{\mho_{n}: n \in N\right\}$. We denote $\mho$ by $\left\{\left[F_{s}, W\left(F_{s}\right)\right]: s \in S\right\}$. For each $F \in \mathscr{F}\left[X^{2}\right]$, there exists exactly one $s \in S$ such that $F \in\left[F_{s}, W\left(F_{s}\right)\right]$. Define $V_{F}=W\left(F_{s}\right)$. Let $\mathfrak{B}=\left\{V_{F}: F \in \mathscr{F}\left[X^{2}\right]\right\}$. It is easily proved that the collection $\mathfrak{B}$ satisfies the condition (c) of Theorem 1. Hence $\mathscr{F}\left[X^{2}\right]$ is paracompact.

Next we consider the hereditarily paracompact case. Suppose that $\mathscr{F}[X]$ is hereditarily paracompact. Then there exists a collection $\mathfrak{U}=\left\{U_{F}: F \in \mathscr{F}[X]\right\}$ of open subsets of $X$ satisfying the condition (c) of Theorem 2. For each $F=\left\{\left(x_{1}\right.\right.$, $\left.\left.y_{1}\right), \ldots,\left(x_{n}, y_{n}\right)\right\} \in \mathscr{F}\left[X^{2}\right]$, where $n=\operatorname{card}(F)$, let $\tilde{F}=\left\{x_{1}, \ldots, x_{n}, y_{1}, \ldots, y_{n}\right\}$. For each $z \in \tilde{F}$, let $\tilde{F}_{z}=\tilde{F}-\{z\}$. For each $z \in \tilde{F}$, let $W_{F}(z)=U_{\tilde{F}}-\tilde{F}_{z}$. Then $U_{z \in \tilde{F}} W_{F}(z)=U_{\tilde{F}}$. For each $F=\left\{\left(x_{1}, y_{1}\right), \ldots,\left(x_{n}, y_{n}\right)\right\} \in \mathscr{F}\left[X^{2}\right]$, where $n=$ $\operatorname{card}(F)$, let $W(F)=\cup_{i=1}^{n} W_{F}\left(x_{i}\right) \times W_{F}\left(y_{i}\right)$. Let $\mathfrak{B}=\left\{W(F): F \in \mathscr{F}\left[X^{2}\right]\right\}$. It suffices to prove that the collection $\mathfrak{B}$ satisfies the condition (c) of Theorem 2. Suppose that $F \subset W(H)$ and $H \subset W(F)$ for some $F=\left\{\left(x_{1}, y_{1}\right), \ldots,\left(x_{n}, y_{n}\right)\right\}$, $H=\left\{\left(z_{1}, w_{1}\right), \ldots,\left(z_{m}, w_{m}\right)\right\} \in \mathscr{F}\left[X^{2}\right]$, where $n=\operatorname{card}(F)$ and $m=\operatorname{card}(H)$. Then $\tilde{F} \subset \bigcup_{q \in \tilde{H}} W_{H}(q)\left(=U_{\tilde{H}}\right)$ and $\tilde{H} \subset U_{p \in \tilde{F}} W_{F}(p)\left(=U_{\tilde{F}}\right)$. By the property of $\mathfrak{u}, \tilde{F} \subset \tilde{H}$ or $\tilde{H} \subset \tilde{F}$. Without loss of generality, we can suppose that $\tilde{F} \subset \tilde{H}$. Since $F \subset W(H)$, we can suppose that $\left(x_{1}, y_{1}\right) \in W_{H}\left(z_{1}\right) \times W_{H}\left(w_{1}\right)$ (if necessary, by reordering the $\left(z_{j}, w_{j}\right)$ 's). Suppose that $x_{1} \neq z_{1}$. Since $\tilde{F} \subset \tilde{H}, x_{1} \in \tilde{H}$. By the definition of $W_{H}\left(z_{1}\right), W_{H}\left(z_{1}\right) \boxplus x_{1}$. But $\left(x_{1}, y_{1}\right) \in W_{H}\left(z_{1}\right) \times W_{H}\left(w_{1}\right)$. This contradiction shows that $x_{1}=z_{1}$. Similarly $y_{1}=w_{1}$. Hence $\left(x_{1}, y_{1}\right)=\left(z_{1}, w_{1}\right)$. Similarly we obtain $\left(x_{i}, y_{i}\right)=\left(z_{i}, w_{i}\right)$ for each $i=2, \ldots, n$ (if necessary, by reordering the $\left(z_{j}, w_{j}\right)$ s, $\left.j=2, \ldots, m\right)$. Hence $F \subset H$. Hence $\mathfrak{B}$ satisfies the condition (c) of Theorem 2 . Hence $\mathscr{F}\left[X^{2}\right]$ is hereditarily paracompact.

\section{Applications.}

TheOREM 4. Suppose that $\mathscr{F}[X]$ and $\mathscr{F}[Y]$ are paracompact. Then $\mathscr{F}[X \times Y]$ is paracompact.

Proor. In the proof of Theorem 3, we in fact prove this theorem. We will give another proof. Let $Z=X \oplus Y$. By Proposition 3.1 in [6], $\mathscr{F}[Z]$ is paracompact. Hence $\mathscr{F}\left[Z^{2}\right]$ is paracompact by Theorem 3. Since $X \times Y$ is a subspace of $Z^{2}$, $\mathscr{F}[X \times Y]$ is a closed subspace of $\mathscr{F}\left[Z^{2}\right]$ (see Proposition 1.2 in [ஞ]). Hence $\mathscr{F}[X \times Y]$ is paracompact.

Remark 1. There exist spaces $X$ and $Y$ such that $\mathscr{F}[X]$ and $\mathscr{F}[Y]$ are hereditarily paracompact, but $\mathscr{F}[X \oplus Y]$ and $\mathscr{F}[X \times Y]$ are not (see [1, Theorem 2.5], [4, Theorem 2.8], and [6, Example 6.6]). 
THEOREM 5. The following conditions are equivalent.

(a) $\mathscr{F}[X]$ is paracompact;

(b) $\mathscr{F}\left[X^{2}\right]$ is paracompact;

(c) $\mathscr{F}\left[X^{m}\right]$ is paracompact for each $m \in N$;

(d) $\mathscr{F}\left[X^{m}\right]^{n}$ is paracompact for each $m, n \in N$.

Proof. By Theorem 1, conditions (c) and (d) are equivalent. The implication (c) $\rightarrow$ (b) is obvious.

(b) $\rightarrow$ (a). Since $X^{2}$ contains a copy of $X$, we can consider $\mathscr{F}[X]$ a closed subspace of $\mathscr{F}\left[X^{2}\right]$ (see Proposition 1.2 in [6]). Hence this implication follows obviously.

(a) $\rightarrow$ (c). Suppose that $\mathscr{F}[X]$ is paracompact. By Theorem 4, it is easily proved by induction that $\mathscr{F}\left[X^{m}\right]$ is paracompact for each $m \in N$.

REMARK 2. Let $D$ be a two point discrete space. Clearly $\mathscr{F}[D]$ is paracompact. But $\mathscr{F}\left[D^{\omega}\right]$ is not normal, where $\omega$ is the first infinite ordinal (see [7]). Therefore we obtain that Theorem 5 cannot be sharpened to assert that $\mathscr{F}\left[X^{\omega}\right]$ is paracompact.

THEOREM 6. The following conditions are equivalent.

(a) $\mathscr{F}[X]$ is hereditarily paracompact;

(b) $\mathscr{F}\left[X^{2}\right]$ is hereditarily paracompact;

(c) $\mathscr{F}\left[X^{m}\right]$ is hereditarily paracompact for each $m \in N$;

(d) $\mathscr{F}\left[X^{m}\right]^{n}$ is hereditarily paracompact for each $m, n \in N$.

Proof. It suffices to prove the implication (a) $\rightarrow(c)$. Suppose that $\mathscr{F}[X]$ is hereditarily paracompact. By Theorem $3, \mathscr{F}\left[X^{2^{n}}\right]$ is hereditarily paracompact for each $n \in N$. For each $m \in N$, there exists an $n \in N$ such that $m<2^{n}$. Since $X^{2^{n}}$ contains a copy of $X^{m}$, we can consider $\mathscr{F}\left[X^{m}\right]$ a closed subspace of $\mathscr{F}\left[X^{2^{n}}\right]$ (see Proposition 1.2 in $[\mathscr{6})$. Hence $\mathscr{F}\left[X^{m}\right]$ is hereditarily paracompact. Hence the implication (a) $\rightarrow$ (c) follows.

By Theorems 4 and 5, we obtain the following.

TheOREM 7. Let $\left\{X_{i}: i=1, \ldots, s\right\}$ be a finite collection of spaces such that $\mathscr{F}\left[X_{i}\right]$ is paracompact for each $i=1, \ldots, s$. Then $\mathscr{F}\left[X_{1}^{m_{1}} \times \cdots \times X_{s}^{m_{3}}\right]^{n}$ is paracompact for $m_{1}, \ldots, m_{s}, n \in N$.

E. K. van Douwen proved that $\mathscr{F}[X]$ is a Moore space if and only if $X$ is first countable [2, Proposition 2.5]. It is well known that for a paracompact Hausdorff space $X, X$ is metrizable if and only if $X$ is a Moore space (see [3, 5.4.1]). Therefore we obtain the following.

THEOREM 8. The following conditions are equivalent.

(a) $\mathscr{F}[X]$ is metrizable;

(b) $\mathscr{F}\left[X^{2}\right]$ is metrizable;

(c) $\mathscr{F}\left[X^{m}\right]$ is metrizable for each $m \in N$;

(d) $\mathscr{F}\left[X^{m}\right]^{n}$ is metrizable for each $m, n \in N$.

THEOREM 9. Let $\left\{X_{i}: i=1, \ldots, s\right\}$ be a finite collection of spaces such that $\mathscr{F}\left[X_{i}\right]$ is metrizable for each $i=1, \ldots$, s. Then $\mathscr{F}\left[X_{1}^{m_{1}} \times \cdots \times X_{s}^{m_{3}}\right]^{n}$ is metrizable for $m_{1}, \ldots, m_{s}, n \in N$. 


\section{REFERENCES}

1. H. Bennet, W. Fleissner and D. Lutzer, Ultraparacompactness in certain Pixley-Roy hyperspaces, Fund. Math. 111 (1980), 11-18.

2. E. K. van Douwen, The Pixley-Roy topology on spaces of subsets, Set Theoretic Topology (G. M. Reed, editor), Academic Press, New York, 1977, pp. 111-134.

3. R. Engelking, General topology, Polish Scientific Publishers, Warsaw, 1977.

4. D. Lutzer, Pixley-Roy topology, Topology Proc. 3 (1978), 139-158.

5. C. Pixley and P. Roy, Uncompletable Moore spaces, Proc. Auburn Topology. Conf. (Auburn, Ala., 1969), Auburn Univ., Auburn, Ala., 1969, pp. 78-85.

6. T. Przymusinski, Normality and paracompactness of Pixley-Roy hyperspaces, Fund. Math. (to appear).

7. M. E. Rudin, Pixley-Roy and the Souslin line, Proc. Amer. Math. Soc. 74 (1979), 128-134.

8. H. Tanaka, Paracompactness of Pixley-Roy hyperspaces. I, Proc. Amer. Math. Soc. 85 (1982), 108-112.

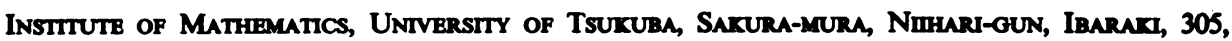
JAPAN 